\title{
Frecuencia serológica de Ehrlichia canis en caninos sospechosos de ehrlichiosis en los distritos de Lima Norte, Perú
}

\author{
Serological frequency of Ehrlichia canis in canines suspected of ehrlichiosis \\ in the northern districts of Lima, Peru
}

Juan Cusicanqui S., ${ }^{1,3}$ Renato Zúñiga F. ${ }^{1,2}$

\section{Resumen}

\begin{abstract}
El estudio tuvo como objetivo determinar la frecuencia serológica de Ehrlichia canis en pacientes caninos en la zona norte de Lima Metropolitana, Perú. Se utilizó la base de datos de un laboratorio de análisis clínicos, el cual recibe muestras de clínicas veterinarias de Lima Norte. Se tomaron los registros que tuvieran análisis contra E. canis durante el periodo 2014-2016 y además, aquellos con análisis de hemograma. Las muestras fueron analizadas con un kit comercial específico para E. canis. Se determinó la frecuencia serológica de E. canis y la asociación entre las variables sexo, edad y raza con los resultados a la prueba. Se halló una frecuencia de 59.4\% (723/1216) de caninos positivos a ehrlichiosis. Se encontró asociación significativa de ehrlichiosis para los pacientes de raza mestiza y de edad mayor a 2 años. En perros adultos, aquellos positivos a la enfermedad presentaron valores menores de la serie roja, blanca y plaquetaria con respecto los perros negativos.
\end{abstract}

Palabras clave: Ehrlichia canis, serología, inmunocromatografía, perros

\section{AbSTRACT}

The study aimed to determine the serological frequency of Ehrlichia canis in canine patients in the northern area of Metropolitan Lima, Peru. Data was obtained from a clinical analysis laboratory, which receives samples from veterinary clinics of the area. Records with analyses against $E$. canis during the 2014-2016 period were taken, as well as

${ }^{1}$ Laboratorio de Análisis Clínico Vet Support, Lima, Perú

${ }^{2}$ Laboratorio de Patología Clínica Veterinaria, Facultad de Medicina Veterinaria y Zootecnia, Universidad Peruana Cayetano Heredia, Lima, Perú

${ }^{3}$ E-mail: juan.cusicanqui.s@upch.pe

Recibido:8 de agosto de 2019

Aceptado para publicación: 14 de mayo de 2020

Publicado: 11 de agosto de 2020 
those with blood count analysis. The samples were analysed with a commercial kit specific for $E$. canis. The serological frequency of $E$. canis and the association between the serological results with the variables sex, age and breed type were evaluated. A frequency of 59.4\% (723/1216) of ehrlichiosis positive canines was found. A significant association of ehrlichiosis was found for patients older than 2 years and for crossbreds. In adult dogs, those positive for the disease have lower values of the red, white and platelet series compared to negative dogs.

Key words: Ehrlichia canis, serology, immunochromatography, dogs

\section{INTRODUCCIÓN}

La ehrlichiosis es una enfermedad causada por una bacteria gram negativa del orden Rickettsiales, género Ehrlichia y se la describe como pleomórfica e intracelular obligada (CFSPH, 2013; Silva et al., 2014). En el género Ehrlichia hay cinco especies reconocidas: E. canis, E. chaffensis, E. ewingii, E. muris y E. ruminantium. De estas, E. canis causa la ehrlichiosis monocítica canina (CFSPH, 2013).

El principal vector para la transmisión de E. canis es la garrapata marrón del perro (Rhipicephalus sanguineus), la cual se infecta al ingerir sangre de un animal infectado (Chávez, 2014). Los perros infectados presentan una fase aguda, con una variedad de signos clínicos inespecíficos, tales como fiebre, letargia, anorexia, y signos gastrointestinales, respiratorios, oculares y neurológicos (Waner y Harrus, 2013). Algunos perros que poseen una inmunidad adecuada o con infecciones leves, pueden superar la fase aguda de la ehrlichiosis y llegar a eliminar a la bacteria sin tratamiento. Sin embargo, otros progresan a la fase subclínica, donde son asintomáticos o con signos clínicos leves, pero también pueden desarrollar la enfermedad crónica, con signos clínicos similares a los de la fase aguda, pero más severos, pudiendo afectar los tejidos hepático, renal y hematopoyético (Mylonakis et al., 2010).
La ehrlichiosis se diagnostica por métodos indirectos como la inmunofluorescencia indirecta (IFI), ELISA e inmunocromatografía (Brandao et al., 2015), o métodos directos como el frotis directo y la reacción en cadena de la polimerasa (PCR) (Cartagena, 2015). Los sistemas inmunocromatográficos se basan en la captura inmunológica de un coloide coloreado durante su paso a través de una membrana en la cual se ha inmovilizado un anticuerpo o antígeno (Acosta, 2003). La aparición de una línea rojo púrpura en la prueba indica la presencia de anticuerpos (Farrell, 2009).

La determinación serológica de anticuerpos contra $E$. canis no solo permite un mejor acercamiento diagnóstico (ya que confirman que el canino ha estado expuesto a la enfermedad); sino que, permite realizar la serovigilancia del estado epidemiológico de la enfermedad. Los kits inmunocromatográficos para la detección de anticuerpos contra E. canis poseen una alta sensibilidad y especificidad en comparación con la prueba de IFI, que es considerada como la prueba de oro.

En el Perú, la ehrlichiosis canina fue detectada en caninos a partir de 1982, y desde ahí se ha incrementado el número de casos reportados (Huerto y Dámaso, 2015). Varios autores han estudiado la enfermedad y su epidemiología, así Carpio (2008) y Aguirre reportaron prevalencias de $70 \%$ en caninos de Máncora y Chosica, respectiva- 
mente, Velásquez (2008) encontró $21 \%$ en perros de la Reserva Nacional de Paracas, Jara (2013) halló 23\% de perros infectados en clínicas veterinarias de Chimbote, y Huerto y Dámaso (2015) reportaron $51.3 \%$ positivos en Huánuco. Por otro lado, en Lima Metropolitana se han reportado seroprevalencias entre 9 y $20 \%$, dependiendo del distrito (Adrianzen et al., 2003).

Se tiene evidencia que al menos el $30 \%$ de la población canina en los distritos de la zona de Lima Norte presentan garrapatas (Estares et al., 2000). Además de ello, la población canina ha ido en aumento, lo que se demuestra en estudios realizados por Arauco et al. (2014) y Soriano et al. (2017) que determinaron 82794 de canes con dueño en el distrito de San Martin de Porres y 85934 canes para el distrito de Comas. A esto se suma la alta cantidad de perros deambulantes (Ochoa et al., 2013).

El objetivo de este estudio fue determinar la frecuencia serológica de E. canis a partir de resultados de un kit inmunocromatográfico en muestras de pacientes caninos remitidas a un laboratorio de la zona norte de Lima en el periodo 2014-2016. Asimismo, evaluar la asociación entre las variables sexo, edad y raza con la seropositividad y la asociación entre los resultados serológicos y los hallazgos hematológicos.

\section{Materiales y Métodos}

El estudio se realizó en el laboratorio de análisis clínico veterinario Vet Support, que recibe muestras remitidas por clínicas veterinarias de los distritos de Carabayllo, Los Olivos, Comas, San Martin de Porres, Independencia y Puente Piedra de Lima Norte.

El estudio fue de tipo observacional y analítico. La población objetivo fueron los registros completos de pacientes entre 2014 y 2016, en los cuales se realizó serología de anticuerpos contra $E$. canis, utilizando el kit comercial Anigen Rapid E.canis Ab Test Kit (Bionote). El kit presenta una alta sensibilidad (97.6\%) y especificidad (99\%) en comparación con la prueba de IFI (Bélanger et al., 2002). Además, se recopiló la información de los análisis hematológicos realizados y se utilizaron los valores de referencia del laboratorio, tanto para el grupo de perros adultos (mayores de cinco meses) como de cachorros (hasta cinco meses).

Se determinó la frecuencia de resultados positivos en base al total de muestras analizadas para cada año. Se elaboraron tablas de estadística descriptiva y se utilizó la prueba de Chi cuadrado para determinar asociación entre el resultado de la prueba y las variables raza y sexo. Para analizar la variable edad se usó prueba de Chi cuadrado y de t de Student. Para los registros que tuvieron análisis hematológicos anexos se elaboró una tabla de resumen descriptivo según resultado a la prueba inmunocromatográfica (positivo y negativo), en la que se indican los valores mínimos, máximo, media y desviación estándar para la serie roja, blanca y plaquetaria. La distribución normal de los datos de las variables cuantitativas continuas se evaluó mediante la prueba de Kolmogorov Smirnov de una muestra. Los datos que siguieron la distribución normal fueron evaluados mediante la prueba de $t$ de Student y para los demás se utilizó la prueba de U de Mann Whitney.

El estudio fue aprobado por el Comité Institucional de Ética para el Uso de Animales (CIEA) de la Universidad Peruana Cayetano Heredia (UPCH). Solo tuvieron acceso a la información generada durante el trabajo el investigador principal, el asesor y los revisores.

\section{Resultados}

Se obtuvieron 1216 registros con orden de serología para investigación de la presencia de anticuerpos para E. canis. De estos, 
Cuadro 1. Resultado a la prueba serológica para detectar Ehrichia canis en perros de Lima Norte, según edad

\begin{tabular}{lccc}
\hline Edad & Pos & Neg & Total \\
\hline 0 - 5 meses & 51 & 55 & 106 \\
5 m - 1.9 años & 174 & 134 & 308 \\
2 - 3.9 -años & 138 & 80 & 218 \\
$4-5.9$-años & 95 & 37 & 132 \\
$6-7.9$-años & 51 & 27 & 78 \\
$8-9.9$-años & 33 & 27 & 60 \\
$10-11.9$-años & 27 & 10 & 37 \\
$12-13.9$-años & 10 & 7 & 17 \\
$14-16$-años & 7 & 1 & 8 \\
Sin datos & 137 & 115 & 252 \\
\hline Total & 723 & 493 & 1216 \\
\hline
\end{tabular}

768 registros incluyeron los datos del hemograma. Se hallaron 68 muestras de cachorros y 557 de adultos; asimismo, 143 no tenían información sobre la edad, por lo que se excluyeron para el análisis de las variables hematológicas.

La frecuencia de casos con serología positiva a $E$. canis fue de $59.4 \%(723 / 1216)$ (Cuadro 1). Se encontró asociación estadística mediante la prueba de Chi cuadrado entre la presencia de anticuerpos contra $E$. canis y la edad en perros mayores a dos años $(p<0.05)$. Los perros positivos tienen una media de edad de $3.5 \pm 3.2$ y los negativos $3.0 \pm 3.0$, diferencia estadísticamente significativa $(p<0.05)$. La prueba de Chi cuadrado indicó una asociación significativa entre raza y la seropositividad $(p<0.05)$, pero no con el sexo de los animales (Cuadro 2).

De los registros con hemogramas $(\mathrm{n}=$ 768), 68 corresponden a cachorros. En el Cuadro 3 se describe los resultados obtenidos para los valores hematológicos con respecto al resultado del kit para anticuerpos de
E. canis en cachorros. Se encontró diferencia significativa entre positivos y negativos a E. canis para los valores de hematocrito, glóbulos rojos y plaquetas $(\mathrm{p}<0.05)$, mientras que para las variables hemoglobina y glóbulos blancos no hubo diferencia estadística significativa. Por otro lado, en perros adultos se encontró diferencia significativa en los valores de hematocrito, hemoglobina, glóbulos rojos, glóbulos blancos y plaquetas $(\mathrm{p}<0.05)$ entre positivos y negativos a E. canis (Cuadro 4).

\section{Discusión}

Si bien se halló que el $59.4 \%$ de las muestras resultaron positivos a E. canis, es posible que este valor sea muy diferente si se incluyen los perros con dueños que no son llevados a veterinarias y perros vagabundos que pueden portar la enfermedad. Se debe considerar, además, que el resultado corresponde a muestras analizadas provenientes de clínicas veterinarias cuyos pacientes tenían sospecha o diagnóstico presuntivo de ehrlichiosis.

Se debe tener en cuenta que los anticuerpos son producto de la inmunidad adquirida de tipo humoral, producidos por las células plasmáticas después de la estimulación del linfocito B (Vega, 2008). Los perros positivos no necesariamente desarrollan la enfermedad, pero han sido expuestos a la enfermedad (McBride et al., 2003). Por lo tanto, una alta frecuencia de anticuerpos contra $E$. canis significa una alta frecuencia de exposición al agente patógeno en los perros llevados a veterinarias en Lima norte.

La frecuencia encontrada puede deberse a la gran cantidad de perros vagabundos en los distritos de Lima Norte (Arauco et al., 2014; Soriano et al., 2017). Estos animales representan una fuente importante del vector ya que, al estar en contacto con otros perros en ambientes comunes tales como jardines y parques, aumentan el riesgo de contagio por el vector (Huerto y Dámaso, 2015) 
Cuadro 2. Resultados serológicos de la prueba de inmunocromatografía para la detección de Ehrlichia canis en perros de Lima Norte (2014-2016), según raza y sexo

\begin{tabular}{clcccc}
\hline \multirow{2}{*}{ Perros } & \multirow{2}{*}{\begin{tabular}{c} 
Total \\
\cline { 3 - 4 }
\end{tabular}} & $(\mathrm{n})$ & \multicolumn{2}{c}{ Resultado } & Porcentaje de \\
& & 588 & 335 & 253 & $57.0^{\mathrm{a}}$ \\
positivo (n) & Negativo (n) & Posidad (\%) \\
\hline \multirow{2}{*}{ Raza } & De raza & 427 & 270 & 157 & $63.2^{\mathrm{b}}$ \\
& Mestizo & 201 & 118 & 83 & 58.7 \\
& No menciona & 628 & 381 & 247 & $60.7^{\mathrm{a}}$ \\
Sexo & Macho & 479 & 279 & 200 & $58.2^{\mathrm{a}}$ \\
& Hembra & 109 & 63 & 46 & 57.8 \\
\hline Total & No menciona & 1216 & 723 & 493 & 59.4 \\
\hline
\end{tabular}

a,b Diferentes letras dentro de las variables raza y sexo son significativamente diferentes $(p<0.05)$

Cuadro 3. Valores hematológicos de 68 perros cachorros (hasta cinco meses) con serología para Ehrlichia canis en la zona de Lima Norte (2014-2016)

\begin{tabular}{llll}
\hline \multirow{2}{*}{ Hemograma } & \multicolumn{2}{c}{ E. canis } & $\begin{array}{l}\text { Rango de } \\
\text { referencia }\end{array}$ \\
\cline { 2 - 4 } & Positivo & Negativo & $29-34 \%$ \\
\hline Hematocrito & $24 \pm 11.8^{\mathrm{a}}$ & $29.7 \pm 9.4^{\mathrm{b}}$ & $9.4-11.2 \mathrm{~g} / \mathrm{dl}$ \\
Hemoglobina & $7.8 \pm 4^{\mathrm{a}}$ & $9.5 \pm 3.3^{\mathrm{a}}$ & $4.3-5.1 \times 10^{6} / \mu 1$ \\
Glóbulos rojos & $3.54 \times 0^{6} \pm 1.87 \times 10^{6} \mathrm{a}$ & $4.36 \times 10^{6} \pm 1.36 \times 10^{6} \mathrm{~b}$ & $4.500-20,000 / \mu 1$ \\
Leucocitos & $10,719 \pm 5,549^{\mathrm{a}}$ & $9,845 \pm 4,517^{\mathrm{a}}$ & $11,300-12,000 / \mu 1$ \\
Neutrófilos & $7,272 \pm 3,970$ & $6,885 \pm 3,457$ & $5,600-12$ \\
N. Abastonados & $86.7 \pm 206.6$ & $200.2 \pm 535.5$ & $0-600 / \mu 1$ \\
N. Segmentados & $7,164 \pm 3,949$ & $6,685 \pm 3,162$ & $5,600-11,400 / \mu 1$ \\
Linfocitos & $3,023 \pm 2,541$ & $2,404 \pm 1,550$ & $3,500-6,500 / \mu 1$ \\
Monocitos & $573.9 \pm 481.2$ & $671 \pm 642.7$ & $700-2,100 / \mu 1$ \\
Eosinófilos & $187.6 \pm 539.7$ & $140.5 \pm 228.9$ & $0-800 / \mu 1$ \\
Basófilos & 0 & 0 & $0-90 / \mu 1$ \\
Plaquetas & $89,262 \pm 80,655^{\mathrm{a}}$ & $176,430 \pm 136,157^{\mathrm{b}}$ & $200,000-410,000$ \\
\hline
\end{tabular}

a,b Diferentes letras dentro de los parámetros hematológicos son significativamente diferentes $(p<0.05)$

${ }^{1}$ Clínica de Animales de Compañía, Universidad Peruana Cayetano Heredia, Lima, Perú 
Cuadro 4. Valores hematológicos de 557 perros adultos (más de cinco meses) con serología para Ehrlichia canis en la zona de Lima Norte (2014-2016)

\begin{tabular}{|c|c|c|c|}
\hline \multirow{2}{*}{ Hemograma } & \multicolumn{2}{|c|}{ E. canis } & \multirow{2}{*}{$\begin{array}{l}\text { Rango de } \\
\text { Referencia }\end{array}$} \\
\hline & Positivo & Negativo & \\
\hline Hematocrito & $34.2 \pm 10.6^{a}$ & $41.1 \pm 10.1^{b}$ & $36-54 \%$ \\
\hline Hemoglobina & $11.3 \pm 3.6^{\mathrm{a}}$ & $13.6 \pm 3.5^{b}$ & $11.8-17.8 \mathrm{~g} / \mathrm{dl}$ \\
\hline Glóbulos rojos & $5 \times 10^{6} \pm 1.61 \times 10^{6} \mathrm{a}$ & $5.97 \times 10^{6} \pm 1.49 \times 10^{6} b$ & $5,200-8,150 \times 10^{6} / \mu 1$ \\
\hline Leucocitos & $10,9583 \pm 7,402^{\mathrm{a}}$ & $13,404 \pm 8,266^{\mathrm{b}}$ & $9,000-15,000 / \mu 1$ \\
\hline Neutrófilos & $8,335 \pm 6,465$ & $10,253 \pm 7,510$ & $4,700-11,550 / \mu$ \\
\hline N. Abastonados & $157.7 \pm 529$ & $232.2 \pm 885$ & $0-225 / \mu 1$ \\
\hline N. Segmentados & $8,176 \pm 6,289$ & $10,018 \pm 7,260$ & $4,500-11,550 / \mu 1$ \\
\hline Linfocitos & $2,017 \pm 1,865$ & $2,051 \pm 1,408$ & $1,350-4,500 / \mu 1$ \\
\hline Monocitos & $651.5 \pm 566$ & $827.5 \pm 769.8$ & $90-750 / \mu 1$ \\
\hline Eosinófilos & $233 \pm 429.3$ & $391.6 \pm 676.3$ & $90-825 / \mu 1$ \\
\hline Basófilos & $1.6 \pm 12.3$ & $12.9 \pm 120.8$ & $0-90 / \mu 1$ \\
\hline Plaquetas & $112,973 \pm 96,823^{a}$ & $191,404 \pm 137,356^{b}$ & $200,000-450,000$ \\
\hline
\end{tabular}

a,b Diferentes letras dentro de los parámetros hematológicos son significativamente diferentes $(p<0.05)$

${ }^{1}$ Clínica de Animales de Compañía, Universidad Peruana Cayetano Heredia, Lima, Perú

El estudio demostró una mayor presencia de anticuerpos contra E. canis en perros mayores de dos años. En este sentido, se ha reportado una mayor frecuencia de ehrlichiosis canina en perros mayores de un año (Asgarali et al., 2012), mayores de 2-4 años (Contreras, 2006) y mayores de 4 años (Jara, 2014) Estos resultados pueden ser explicados debido a que los perros a partir de edad mediana tienen mayor probabilidad de exposición a garrapatas que los cachorros, por la costumbre de los dueños de pasear a sus canes una vez completadas las vacunas (Huerto y Dámaso, 2015).

La asociación significativa entre grupo racial y seropositividad a $E$. canis estuvo de acuerdo con el trabajo de Cartagena et al. (2015), quienes encontraron una mayor prevalencia en perros mestizos; no obstante,
Huerto y Dámaso (2015) no encontraron diferencias significativas entre razas. Por otro lado, no se encontró asociación significativa entre la presencia de anticuerpos contra $E$. canis y el sexo de los caninos, habiendo resultados contradictorios en la literatura; así, Adrianzen et al. (2003) reportan una mayor prevalencia en hembras, mientras que Asgaraly et al. (2012), Contreras (2006) y Cartagena et al. (2015) no encontraron diferencias significativas entre estas variables.

Se ha descrito un comportamiento fisiológico diferente entre perros jóvenes y adultos para los valores eritrocitarios y leucocitarios (Harper et al., 2003; Lawler et al., 2007; Brenten et al., 2016), de allí que en este estudio se analizaron por separado. En el análisis de las variables hematológicas en cachorros, en la serie roja se obtuvo una 
media más baja de hemoglobina y glóbulos rojos en perros positivos a la enfermedad con respecto a los negativos, sin embargo, al analizar la hemoglobina no se encontró diferencia estadística significativa. Hay que tener en cuenta que la significancia para la hemoglobina fue muy cercana a $0.05(\mathrm{p}=0.058)$.

No se encontró estudios similares que comparen la frecuencia o prevalencia de la enfermedad y las variables hematológicas en cachorros. Sin embargo, en los adultos, la serie roja, blanca y plaquetaria presentaron valores promedio más bajos en perros positivos a ehrlichiosis con respecto a los negativos, lo cual concuerda con el estudio de Elitok y Ungur (2016) y Fonseca et al. (2017), aunque Aguirre (2008) no encontró tales diferencias. La trombocitopenia en diferentes grados es el hallazgo hematológico característico en las fases de la enfermedad producida por E. canis (Straube, 2010; Waner y Harrus, 2013). La bacteria se multiplica en las células mononucleares y la trombocitopenia se debería a un mayor consumo, secuestro y destrucción de las plaquetas (López et al., 1999).

Hay que tener en cuenta que, en exposiciones agudas, la presencia de anticuerpos no siempre está presente en el animal infectado (Munhoz et al., 2012). En esta fase de la enfermedad, la trombocitopenia es el hallazgo hematológico más común (RodríguezVivaz et al., 2005). Esto puede explicar los casos de perros negativos a la prueba de anticuerpos contra E. canis, que acudieron a consulta al presentar sintomatología compatible y que presentaron trombocitopenia.

\section{Conclusiones}

- Se confirmó la exposición a Ehrlichia canis en el $54.8 \%$ de perros, cuyas muestras fueron llevadas a un laboratorio de Lima Norte.

- Los animales adultos tienen una mayor probabilidad de haberse expuesto a la enfermedad.
- Los perros diagnosticados como positivos tienen valores hematológicos menores a los negativos.

- El estudio sugiere una alta prevalencia de E. canis en Lima Norte.

\section{Literatura Citada}

1. Acosta M. 2003. Desarrollo y evaluación de una prueba inmunocromatográfica para el diagnóstico de la infección con Tripanosoma cruzi. Tesis de Maestría. Paraguay: Univ. Nacional de Asunción. $95 \mathrm{p}$.

2. Adrianzen J, Chávez A, Casas E, Li O. 2003. Seroprevalencia de la dilofiraliosis y ehrlichiosis canina en tres distritos de Lima. Rev Inv Vet Perú 14: $45-$ 47. doi: 10.15381/rivep.v14i1.1596

3. Aguirre R. 2008. Frecuencia de Ehrlichia canis en caninos de Chosica con cuadro de pancitopenia mediante el uso de PCR. Tesis de Médico Veterinario Zootecnista. Lima: Univ. Peruana Cayetano Heredia. 22 p.

4. Arauco D, Betty U, León D, Falcón N. 2014. Indicadores demográficos y estimación de la población de canes con dueño en el Distrito de San Martin de Porres. Salud Tecnol Vet Perú 2: 83-92. doi: $10.20453 /$ stv.v2i2.2254

5. Asgarali Z, Pargass I, Adamb J, Mutani A, Ezeokoli C. 2012. Haematological parameters in stray dogs seropositive and seronegative to Ehrlichia canis in North Trinidad. Ticks Tick-Borne Dis 3: 207-211. doi: 10.1016/ j.ttbdis.2012.03.006

6. Bélanger M, Sorenson H, France M, Bowie M, Barbet A, Breitschwerdt E, Rick A. 2002. Comparison of serological detection methods for diagnosis of Ehrlichia canis infections in dogs. J Clin Microbiol 40: 3506-3508. doi: 10.1128/ JCM.40.9.3506-3508.2002

7. Brenten T, Morris PJ, Salt C, Raila J, Kohn B, Schweigert FJ, Zentek J. 2016. Age-associated and breed- 
associated variations in haematological and biochemical variables in young labrador retriever and miniature schnauzer dogs. Vet Rec Open 3: e000166. doi: 10.1136/vetreco-2015-000166

8. Carpio L. 2008. Detección de anticuerpos contra Ehrlichia Canis en caninos domesticos infestados con garrapatas en el distrito de Mancora, Piura. Tesis de Médico Veterinario Zootecnista. Lima: Univ. Peruana Cayetano Heredia. 21 p.

9. Cartagena LM, Ríos LA, Cardona JA. 2015. Seroprevalencia de Ehrlichia Canis en perros con sospecha de infeccion por patogenos transmitidos por garrapatas en Medellín, 2012-2014. Rev Med Vet 29: 51-62.

10. [CFSPH] The Center for Food Security and Public Health. 2013. Ehrlichiosis and anaplasmosis: zoonotic species. Iowa, USA: CFSPH. $14 \mathrm{p}$.

11. Chávez C. 2014. Ehrlichia canis en caninos y el tratamiento con doxiciclina.Tesis de Médico Veterinario Zootecnista. Lima: Univ. Nacional Mayor de San Marcos. 99 p.

12. Contreras A. 2006. Estudio retrospectivo de caso control de ehrlichiosis canina en la Facultad de Medicina Veterinaria de la Universidad Mayor de San Marcos: periodo 2002-2005. Tesis de Médico Veterinario. Lima: Univ. Nacional Mayor de San Marcos. 45 p.

13. Elitok B, Ungur B. 2016. Prevalence of Ehrlichia canis infection in Usak and investigation of clinical, hematological and biochemical signs in infected dogs. Int Biol Biomed J 4: 134-139.

14. Estares L, Chávez A, Casas E. 2000. Ectoparasitos en caninos de los distritos de la zona climática norte de Lima Metropolitana. Rev Inv Vet Perú 11: 72-76. doi: 10.15381/rivep.v11i1.6806

15. Farrell B. 2009. Lateral flow immunoassay. Nueva York: Springer. $225 \mathrm{p}$.

16. Fonseca JP, Bruhn F, Ribeiro M, Hirsch C, Rocha C, Guedes E, Guimarães A. 2017. Hematological parameters and seroprevalence of Ehrlichia canis and Babesia vogeli in dogs. Ciênc Anim Bras 18: e-36095. doi: 10.1590/1089-6891v18e-36095

17. Guedes PE, Oliveira TN, Carvalho F, Carlos RS, Albuquerque G, Munhoz A, Wenceslau A, et al. 2015. Canine ehrlichiosis: prevalence and epidemiology in northeast Brasil. Rev Bras Parasitol Vet 24: 115-121. doi: 10.1590/S198429612015030

18. Harper EJ, Hackett RM, Wilkinson J, Heaton PR. 2003. Age-related variations in hematologic and plasma biochemical test results in Beagles and Labrador Retrievers. J Am Vet Med Assoc 223: 1436-1442. doi: 10.2460/ javma.2003.223.1436

19. Huerto-Medina E, Dámaso-Mata B. 2015. Factores asociadas a la infeccion por Ehrlichia canis en perros infestados con garrapatas en la ciudad de Huánuco, Perú. Rev Per Med Exp Salud Pública. 32: 756-760.

20. Jara M. 2014. Frecuencia de Ehrlichia canis en perros en la ciudad de Chimbote - 2013. Tesis de Médico Veterinario. Cajamarca, Perú: Univ. Nacional de Cajamarca. $40 \mathrm{p}$.

21. Lawler DF, Ballam JM, Meadows R, Larson BT, Li Q, Stowe HD, Kealy RD. 2007. Influence of lifetime food restriction on physiological variables in Labrador retriever dogs. Exp Gerontol 42: 204-214. DOI: 10.1016/j.exger.2006.09.010

22. López J, Castillo A, Muñoz M, Hildebrandt S. 1999. Hallazgo de Ehrlichia canis en Chile. Informe preliminar. Arch Med Vet 31: 211-214. doi: 10.4067/S0301-732X1999000200008

23. McBride JW, Corstvet RE, Gaunt SD, Boudreaux C, Guedry T, Walker DH. 2003. Kinetics of antibody response to Ehrlichia canis immunoreactive proteins. Infect Immun 71: 2516-2524. doi: 10.1128/iai.71.5.2516-2524.2003

24. Munhoz TD, Faria JL, VargasHérnandez, G, Fagliari JJ, Santana AE, Machado RZ, Tinucci-Costa M. 2012. Experimental Ehrlichia canis infection changes acute-phase proteins. 
Rev Bras Parasitol V 21: 206-212. doi: 10.1590/s1984-29612012000300006

25. Mylonakis ME, Siarkou VI, Koutinas AF. 2010. Myelosuppressive canine monocityc ehrlichiosis: an update on the pathogenesis, diagnosis and management. Isr J Vet Med 65: 129-135.

26. Ochoa Y, Falcón N, Zuazo J, Guevara B. 2013. Estimacion de la poblacion de perros deambulantes en el distrito de Los Olivos, Lima, Perú. Rev Inv Vet Perú 25: 366-377. doi: 10.15381/rivep.v25i3.-10114

27. Rodriguez-Vivas RI, Albornoz RE, Bolio GM. 2005. Ehrlichia canis in dogs in Yucatan, Mexico: seroprevalence, prevalence of infection and associated factor. Vet Parasitol 127: 7579. doi: 10.1016/j.vetpar.2004.08.022

28. Silva AB, Canseco S, De la Torre, M, Mayoral A, Mayoral M, et al. 2014. Infeccion humana asintomatica por con- tacto con perros. Un caso de ehrlichiosis humana. Gac Med Mex 150: 171-174.

29. Soriano J, Nuñez J, León, D, Falcón, N. 2017. Estimacion de la población de canes con dueño en el distrito de Comas, Lima- Perú. Rev Cienc Vet 33: 5-10.

30. Straube J. 2010. Canine ehrlichiosis From acute infection to chronic disease. CVBD Digest 7: 7-8.

31. Vega G. 2008. La respuesta inmune. Rev Fac Med UNAM 51: 128-129.

32. Velasquez T. 2008. Evidencia serologica de Ehrlichia canis en los caninos domesticos de la Reserva Nacional de Paracas. Tesis de Médico Veterinario Zootecnista. Lima: Univ. Peruana Cayetano Heredia. 16 p.

33. Waner T, Harrus S. 2013. Canine monocytic ehrlichiosis - from pathology to clinical manifestations. Isr J Vet Med 68: 12-18. 